\title{
The Effects of Maximal Intensity Exercise on Cognitive Performance in Children
}

\author{
by \\ Roy David Samuel ${ }^{1}$, Ofir Zavdy², Miriam Levav³, Ronen Reuveny4, Uriel Katz ${ }^{4}$, \\ Gal Dubnov-Raz ${ }^{5,6}$
}

High intensity physical exercise has previously been found to lead to a decline in cognitive performance of adults. The aim of this study was to determine the effects of maximal intensity exercise on cognitive performance of children. Using a repeated-measures design, 20 children and adolescents aged 8-17 years completed a battery of tests measuring memory and attention. Forward and Backward Digit Span tests, the Rey Auditory-Verbal Learning Test (RAVLT) and the Digit Symbol Substitution Test (DSST) were performed at baseline, immediately after, and one hour after a maximal cardiopulmonary exercise test. Forward and Backward Digit Span scores significantly improved postrecovery compared with baseline measurements. There was a significant decrease in RAVLT scores post-exercise, which returned to baseline values after recovery. The DSST test scores were mildly elevated from post-exercise to after recovery. Maximal intensity exercise in children and adolescents may result in both beneficial and detrimental cognitive effects, including transient impairment in verbal learning. Cognitive functions applying short term memory improve following a recovery period. Parents, educators and coaches should consider these changes in memory and attention following high-intensity exercise activities in children.

Key words: neurocognitive, verbal learning, physical activity, short term memory, cognition, pediatrics.

\section{Introduction}

It is well established that physical activity (PA) and planned exercise have significant health benefits in numerous physical, mental and social aspects (Physical Activity Guidelines Advisory Committee, 2008; Strong et al., 2005). A number of observational studies in children have shown a positive correlation between $\mathrm{PA}$, or physical fitness, and academic achievements (Carlson et al., 2008; Singh et al., 2012; Tomporowski, 2003; Tomporowski et al., 2008). Interventional studies

have further demonstrated the effect of a PA program on cognitive performance in children (Davis et al., 2011; Hillman et al., 2014). Such programs typically involve daily moderatevigorous physical activity (MVPA) of 20-60 minutes duration, sufficient to increase cardiorespiratory fitness in children. A meta-analysis of 44 studies found a positive, yet weak, correlation (a general effect size of $d=0.32$ ) between PA and different measures of cognitive performance, such

\footnotetext{
1 - Department of Physical Education and Movement, Kibbutzim College of Education Technology and the Arts, Tel Aviv, Israel, and Department of Sport Management, Florida State University, FL, USA.

2- Hebrew University-Hadassah Medical School, Jerusalem, Israel.

3 - Department of Pediatric Rehabilitation, The Edmond and Lily Safra Children's Hospital, Sheba Medical Center, Tel-Hashomer, Israel.

4. Edmond J. Safra International Congenital Heart Center, The Edmond and Lily Safra Children's Hospital, Sheba Medical Center, Tel-Hashomer, Israel.

5 - Exercise, Nutrition and Lifestyle Clinic, The Edmond and Lily Safra Children's Hospital, Sheba Medical Center, Tel-Hashomer, Israel.

6 - Sackler Faculty of Medicine, Tel Aviv University, Tel Aviv, Israel.
} 
as IQ, academic achievements, math and verbal tests, and perceptual skill tests in children (Sibley and Etnier, 2003). A more recent review of the literature on the effects of PA on children's cognitive function (64 studies) and academic achievements (73 studies) also reported positive effects (Donelly et al., 2016). Specifically, most studies suggested that single bouts of MVPA and participation in PA interventions benefit children's mental functioning.

According to Tomporowski et al. (2011), cognition is a general term reflecting different processes, including perception, attention, memory, working memory, pattern recognition, executive function, concept formation and reasoning, intelligence, and academic achievement. Studies of the relationship between exercise and cognition have used various tests measuring global processing (e.g., intelligence and academic achievement) as well as specific components of cognition (e.g., memory and executive function). These studies indicated that most gains of moderate intensity exercise can be seen in executive functioning. Executive functioning involves cognitive function such as scheduling, planning, response inhibition, working memory, controlled processing, visuospatial processing, and speeded processing (Best, 2010; Tomporowski et al., 2008, 2011). For example, in a trial of 171 sedentary, overweight middle-school children, Davis et al. (2011) demonstrated that $40 \mathrm{~min} /$ day of moderatevigorous intensity aerobic exercise brought about higher scores on a cognitive assessment of planning ability compared with a control sedentary group of children performing only 20 $\mathrm{min}$ /day of activity.

In contrast to the well-established cognitive benefits of regular MVPA in children, the influence of acute high intensity exercise on cognitive performance requires further examination. In a meta-analysis of 79 studies that examined the relationship between exercise and cognition, only nine studies involved children (i.e., 175 effect sizes) with the majority of participants being young and mature adults (Chang et al., 2012). This stresses the need for additional studies in the pediatric age range. Furthermore, this meta-analysis found that several factors may moderate the effects of acute exercise on post-exercise cognitive performance, including exercise intensity, cognitive performance measurement, and time of measurement in relation to exercise (Chang et al., 2012). Specifically, whereas very light to moderate exercise intensity resulted in positive cognitive effects immediately post-exercise, hard to maximal exercise intensity did not produce significant effects. Additionally, the type of cognitive function tested was found to be a moderating factor of the exercise-cognitive performance effect. For example, significant positive effects of acute exercise were reported for free recall and verbal fluency, whereas negative effects were reported for the backwards digit span and the auditory verbal learning test (Chang et al., 2012). It was also reported that the timing of the test administration following exercise posed a significant effect: tests administered within 0-10 min of exercise completion resulted in significant negative effects, whereas the largest positive effects were observed following 11-20 $\mathrm{min}$ of delay, and smaller positive effects evident following 20 min of delay (Chang et al., 2012).

Acute high intensity physical exercise has previously been found to lead to a decline in cognitive performance of adults, measured during exercise (Isaacs and Pohlman, 1991; McMorris and Keen, 1994) or immediately following the exercise bout (Covassin et al., 2007; Lo Bue-Estes et al., 2008; Moore et al., 2012). Covassin et al. (2007) assessed cognitive performance following acute high intensity exercise in 102 recreational adult athletes. All participants completed a 25-min test battery measuring various cognitive functions (e.g., attention, verbal recognition memory, visual working memory) at baseline and again immediately upon completion of a maximal exercise test on a treadmill. Control group participants completed the test again immediately following a $15 \mathrm{~min}$ rest. Three days later, all participants completed a third post-test. There was a significant increase in self-reported fatigue for the exercise group from baseline to postexercise. Also, there was a decrease in verbal memory, immediate recall memory, and delayed recall memory in the exercise group, which returned close to baseline after three days. There were no significant differences in visual memory, motor processing, speed, and reaction time between the experimental and control groups at three measurement points. 
In another study, Lo Bue-Estes et al. (2008) tested healthy and sedentary female college students on selected cognitive variables prior to and directly after a maximal exercise test $(3 \mathrm{~min})$, as well as after 30-min recovery. The results indicated significant decrements in working memory during and immediately after the exercise, that significantly improved after recovery. Therefore, an acute bout of high intensity exercise could pose both debilitating and facilitating effects on cognitive performance, depending on the time of cognitive testing in relation to exercise completion, as well as the type of cognitive function assessed.

The decrease in working memory following maximal exercise might result from vascular changes in the brain and cerebral fatigue. Shibuya et al. (2004a, b) found that supramaximal dynamic exercise in healthy young adults resulted in decreased cerebral oxygenation, which signifies lower cerebral cortex activity. Furthermore, Nishihira et al. (1999) found that in young adults, an elevated heart rate $(180 \mathrm{bpm})$ following highintensity exercise resulted in a lower P300 amplitude (i.e., an index of brain activity required in the maintenance of working memory), indicating a cognitive function decrease. Therefore, it seems that very high intensity exercise affects normal brain functioning that can result in a transient cognitive decline.

Recently, researchers have begun considering the effects of higher intensity exercise on cognition, as an alternative to the traditional MVPA. For example, Alves et al. (2014) performed acute high-intensity interval training (HIIT; 60\%$80 \%$ of the maximal heart rate) in a sample of 22 healthy middle-aged individuals, and found that the HIIT session improved the performance in a Stroop Color Word test that measures selective attention, but not in a Digit Span Forward and Backward tests that measure short-term memory. Yet, the study protocol included a short cool down period from the exercise to the onset of cognitive testing. These authors suggested that exercise intensity may not be the predominant factor affecting cognitive performance, but rather the exercise-induced fatigue. Similarly, Ma et al. (2015) examined the effects of a 2-week intervention of 4-min, high-intensity interval activities named "Funtervals" (e.g., squats, jumping jacks, scissor kicks, jumping, and running on the spot) on selective attention of 88 elementary school children. The results indicated a degree of improvement in selective attention following the exercise intervals, yet no measurements of the heart rate or fatigue were taken, and there was a 10-min delay from the end of the exercise to the onset of the attention test.

Reviewing the literature on the exercisecognition relationship in children, Donnelly et al. (2016) stated that "we are sorely limited in our ability to provide specific direction with regard to the mode, duration, frequency, and intensity of exercise necessary to provide meaningful benefits for cognition" (p. 1217). The results of the HIIT studies (Alves et al., 2014; Ma et al., 2015) suggest that more attention should be devoted to examining the role of intensity and the schedule of exercise in relation to cognitive effects. Collectively, previous studies conducted in adults found transient effects of high intensity exercise on working memory, attention, verbal memory, and immediate recall memory (Covassin et al., 2007; Lo Bue-Estes et al., 2008; Moore et al., 2012). We are unaware of similar studies conducted in children. It appears that high intensity exercise can have both positive and negative effects on children's cognitive function, depending on the time delay from exercise to cognitive activation and the type of cognitive function activated. This could have important clinical implications for educational as well as competitive sport settings. Therefore, the aim of the current study was to examine the effects of a single bout of maximalintensity exercise on cognitive functions in children and adolescents.

\section{Material and Methods}

Study Design

Children and adolescents who were referred for a cardiopulmonary exercise test (CPET) in our medical institution were invited to participate in the study. The participants underwent a series of neuro-cognitive tests before the CPET, immediately after it, and after a one hour rest. The study was approved by the Institutional Review Board of the Sheba Medical Center, Tel-Hashomer, Israel, and conducted according to the Declaration of Helsinki. Each participant gave verbal assent to participate in the study, and at least one parent signed an informed consent form prior to any testing procedure. 


\section{Participants}

Inclusion criteria were age between 8-17 years, willingness to complete all testing procedures, obtaining signed consent forms from parents and obtaining verbal assent from the participants. Exclusion criteria were the presence of any acute disease, complicated congenital heart defects, asthma, obesity, any known psychiatric condition (including attention-deficit/ hyperactivity disorder), or use of any immediate or chronic medications or dietary supplement. Participants were also excluded after the exercise test if they had not achieved maximal exercise intensity, as defined by a clinical appearance of maximal effort (e.g., facial flushing, heavy sweating, labored breathing, intense exertion) and a respiratory exchange ratio (RER) of over 1.05. Anthropometry Measures

Body height was measured using a wallmounted stadiometer (Seca 206, Seca gmbh stadiometer, Hamburg, Germany). Body mass was evaluated using an electronic scale (Tanita BC418-MA, Tokyo, Japan). The BMI was calculated as body mass in $\mathrm{kg}$ divided by the square of stature in meters. Body height, mass and BMI were converted to percentiles according to the United States Centers for Disease Control and Prevention reference curves. Obesity was defined as $\mathrm{BMI} \geq 95^{\text {th }}$ percentile for age and sex.

Cardiopulmonary Exercise Testing (CPET)

The CPET was performed on a cycle ergometer connected to a metabolic system (ZAN 600 CPET: ZAN Messgerate GmbH, Oberthulba, Germany). Tests were performed during the morning hours, no later than noon. The gas exchange system was calibrated each morning prior to testing. The CPET protocol included a ramp increment of 10,15 or 20 watts. $\mathrm{min}^{-1}$, according to the predicted maximal achievable load of each participant (Godfrey, 1974), and ended when the participant could no longer maintain the required cadence of 60 RPM for over $5 \mathrm{~s}$ (i.e., after 8-12 min). Maximal oxygen uptake (peak $\mathrm{VO}_{2}$ ) was the mean volume of oxygen consumed per kilogram of body mass per minute $\left(\mathrm{mL} \cdot \mathrm{kg}^{-1} \cdot \mathrm{min}^{-1}\right)$, during the last $20 \mathrm{~s}$ of the exercise test, expressed in both absolute values and as a percentage of predicted values (Bongers et al., 2014). The maximal workload was the actual maximal load the participant achieved, expressed in both absolute values and as a percentage of predicted values (Bongers et al., 2014). The peak heart rate was the maximal value obtained during the exercise test, expressed both in absolute terms (beats. $\mathrm{min}^{-1}$ ) and as a percentage of predicted values (Bongers et al., 2014).

Neuro-Cognitive Tests

Based on previous research in adults, the anticipated cognitive performance effects following maximal exercise resided in areas of memory and attention (Covassin et al., 2007; Lo Bue-Estes et al., 2008; Moore et al., 2012). We therefore selected tests that measured these domains, and that qualified in a number of criteria: (a) all had to be appropriate for children and adolescents aged 8-17 years old, (b) they had to be in Hebrew, well validated, and to have a high sensitivity in tracking attention impairment, and (c) they had to be easy and quick to perform, as they had to be conducted exactly at exercise termination and after one hour. A battery of four tests was assembled, administered in the following order: (a) Forward and (b) Backward Digit Span (WISC-IV, Wechsler 2003), (c) a variation of the Rey Auditory-Verbal Learning Test (RAVLT, Vakil et al., 1998), and (d) Digit Symbol Substitution Test (WISC-IV, Wechsler, 2003). Each test was preceded by an example or short training section inherent to the testing procedure (e.g., five practice symbols at the beginning of the DSST).

Digit span. WISC-IV (Wechsler, 2003) measures two main components: the attention span and short term memory, evaluated during the first task, and verbal working memory, evaluated during a second, backwards task (Diamond, 2013). This part of memory is involved in learning, language comprehension and shortterm storage of information for executing complex tasks (Wechsler, 2003; Vakil et al., 2009). The participants are verbally presented with a series of digits (e.g., 6, 2, 9, 7) at a rate of one digit per second, and are required to repeat them verbatim. If they succeed, they are given a longer list (e.g., 5, $3,8,1,6)$. The number of digits increases by one until the participant consecutively fails two trials of the same digit span length. The length of the longest list a person can remember is that person's digit span. This test contains two separate tasks. In the "Forward Digit-Span", participants are asked to repeat the digits in the given called order. In the "Backward Digit-Span", participants 
are required to repeat the digits in reverse. In the experiment, the participant received one point for each correct answer. Both stages of the test were scored separately. The maximum number of points in the entire test was 32. Different lists of numbers were given to the participants in each of the three testing occasions, to minimize a learning effect.

Modified Rey auditory-verbal learning test (RAVLT). This test has several parts, and for the purpose of this study, only the first part that evaluates verbal learning ability and immediate recall memory (Vakil et al., 1998) was used. In this test, a list of 15 common nouns (e.g., drum, curtain, bell) was read to the child at a pace of one word per second. The child was then immediately asked to repeat as many of the 15 words as he or she remembered. The test had no time limit, and was performed once due to the anticipated rapidly transient nature of the expected cognitive decline. Different words of a similar length and context were given to the participants in each of the three testing occasions, in order to minimize a learning effect.

Digit symbol substitution test (DSST). The DSST assesses visual-motor coordination, short term memory, concentration and processing speed, along with the ability to learn a new task (Wechsler, 2003; Vakil et al., 2009). These abilities are needed in sport (eye-hand/leg coordination), art (writing, drawing, sculpting), and even in daily activities, such as tying shoes and using scissors. In this test, the child matches simple geometric symbols with corresponding digits using a key of symbols. The task is to achieve as much correct signs as possible in 120 seconds. Each correct symbol awards the participant with 1 point. The score was the sum of all correct symbols matched by the child. Different combinations of symbols and digits were given to the participants in each of their three testing occasions, to minimize a learning effect.

\section{Statistical Analyses}

The sample size was calculated based on the expected change in cognitive performance from baseline to immediately after the exercise using WinPepi version 11.42. Based on previous works (Covassin et al., 2007; Lo Bue-Estes et al., 2008; Moore et al., 2012), we hypothesized a $10 \%$ decline in cognitive functions measured by the neuro-cognitive tests and a standard deviation of $15 \%$. Therefore, with significance set at $5 \%$ and power at $80 \%$, 16 children were needed for the study.

Normal distribution was verified using the Shapiro-Wilk test. The Pearson's correlation coefficient was used to test the relationship between the fitness level and various test scores. The test scores of each neuro-cognitive test were compared between the three time points using the one-way repeated measures analysis of variance with adjustment for least square differences and post-hoc testing to identify significant pairwise differences in test scores between the three time points. Partial eta squared $\left(\eta^{2}\right)$ was used as a measure of effect size. The level of statistical significance was set at $p=0.05$.

\section{Results}

Twenty children (12 males, 8 females) that fulfilled all inclusion and exclusion criteria were included in the study. Their clinical characteristics and exercise test data are presented in Table 1. The selected participants completed all tests and performed maximal effort on the exercise test.

The duration of the test battery, including explanations and training, was less than 30 minutes: $\sim 10$ minutes needed for each digit span test, $\sim 5$ minutes for the RAVLT and $\sim 5$ minutes for the DSST. Neuro-cognitive testing mean scores, as well as the norms for each test used for boys and girls in the relevant age range for this study (mean age of 13 yrs), are specified in Table 2. All test scores were comparable to the predicted normal scores, with differences of up to $10 \%$. No significant associations were found between the baseline scores of the tests used. There were no significant correlations between aerobic fitness, expressed as the percentage of predicted values, and digit span, RAVLT or DSST scores.

Digit Span

The mean scores of the forward and backward digit span tests are presented in Figure 1 (A and B, respectively). The mean test scores in the forward digit span test differed significantly across time (Wilk's Lambda $=0.67, F(2,18)=4.4, p$ $=0.028, \eta^{2}=0.33$ ). Post-hoc analyses revealed that the mean test scores were significantly higher after the one-hour recovery from the exercise test as compared with baseline values $(p=0.02)$.

The mean test scores in the backwards digit span test also differed significantly across time $($ Wilk's Lambda $=0.33, F(2,18)=18.2, p<$ 
0.001, $\left.\eta^{2}=0.67\right)$. Post-hoc analyses revealed that the mean test scores were significantly higher after the recovery period both as compared with baseline $(p=0.01)$ and immediate post-exercise values $(p<0.001)$.

RAVLT

The mean scores of the RAVLT are presented in Figure 2A. Mean test scores differed significantly across time (Wilk's Lambda $=0.38$, $\left.F(2,18)=14.7, p<0.001, \eta^{2}=0.62\right)$. Post-hoc paired analyses revealed a significant decrease in test scores from baseline to post-exercise $(p=0.02)$ and a significant increase from post-exercise to the one-hour recovery measurement $(p<0.001)$. There was no significant difference in mean RAVLT test scores between baseline and one-hour recovery ( $p$ $=1.00)$.

DSST

The mean scores of the DSST are presented in Figure 2B. Mean test scores differed significantly across time (Wilk's Lambda $=0.67, F(2,18)=4.4, p$ $\left.=0.029, \eta^{2}=0.33\right)$. Post-hoc paired analyses revealed a significant difference only between post-exercise and recovery measurements ( $p=$ $0.01)$. There was no significant difference in mean test scores between baseline and one-hour recovery $(p=0.34)$.

\section{Table 1}

Clinical characteristics and exercise performance of study participants.

Data are presented as Mean (SD)

\begin{tabular}{|c|c|}
\hline Clinical Characteristic & $\begin{array}{l}\text { All participants } \\
(\mathrm{n}=20)\end{array}$ \\
\hline Age (years) & $13.1(2.4)$ \\
\hline Body mass & \\
\hline $\mathrm{kg}$ & 49.5 (14.1) \\
\hline percentile & $52.3(32.3)$ \\
\hline Body height & \\
\hline $\mathrm{cm}$ & 156.7 (11.3) \\
\hline percentile & 60.5 (27.4) \\
\hline Body mass index & \\
\hline $\mathrm{kg} / \mathrm{m}^{2}$ & $19.8(4.0)$ \\
\hline percentile & $55.8(29.8)$ \\
\hline $\begin{array}{l}\text { Exercise test duration } \\
\text { min:sec }\end{array}$ & 10:15 (01:05) \\
\hline $\begin{array}{l}\text { Peak heart rate } \\
\text { beats } \mathrm{min}^{-1}\end{array}$ & $186(12)$ \\
\hline$\%$ predicted peak & $98(7)$ \\
\hline Peak load & \\
\hline Watt & $137(79)$ \\
\hline$\%$ predicted & $86(20)$ \\
\hline $\begin{array}{c}\text { Peak } \mathrm{VO}_{2} \\
\mathrm{~mL} \cdot \mathrm{kg}^{-1} \cdot \mathrm{min}^{-1}\end{array}$ & $41.3(10.4)$ \\
\hline$\%$ predicted & $94(19)$ \\
\hline Peak RER & $1.17(0.09)$ \\
\hline
\end{tabular}


Table 2

Hebrew test norms for age 13 and baseline scores in the present study for the Forward and Backward Digit Span Test, Rey Auditory-Verbal Learning Test (RAVLT), and Digit Symbol Substitution Test (DSST). Data are presented as Mean (SD)

\begin{tabular}{lllll}
\hline Neuro-cognitive test & $\begin{array}{l}\text { Boys - Norms } \\
M(S D)\end{array}$ & $\begin{array}{l}\text { Boys - Study } \\
\text { Scores } \\
M(S D)\end{array}$ & $\begin{array}{l}\text { Girls - Norms } \\
M(S D)\end{array}$ & $\begin{array}{l}\text { Girls - Study } \\
\text { Scores } \\
M(S D)\end{array}$ \\
\hline Forward Digit Span & $8.34(2.41)$ & $8.80(2.00)$ & $7.70(1.91)$ & $7.40(1.10)$ \\
Backward Digit Span & $6.07(2.15)$ & $5.50(1.70)$ & $5.48(1.60)$ & $6.50(1.20)$ \\
RAVLT & $7.29(1.50)$ & $7.80(0.90)$ & $7.10(1.73)$ & $8.60(2.20)$ \\
DSST & $55.78(9.28)$ & $59.80(19.20)$ & $59.10(11.83)$ & $64.30(7.50)$ \\
\hline
\end{tabular}

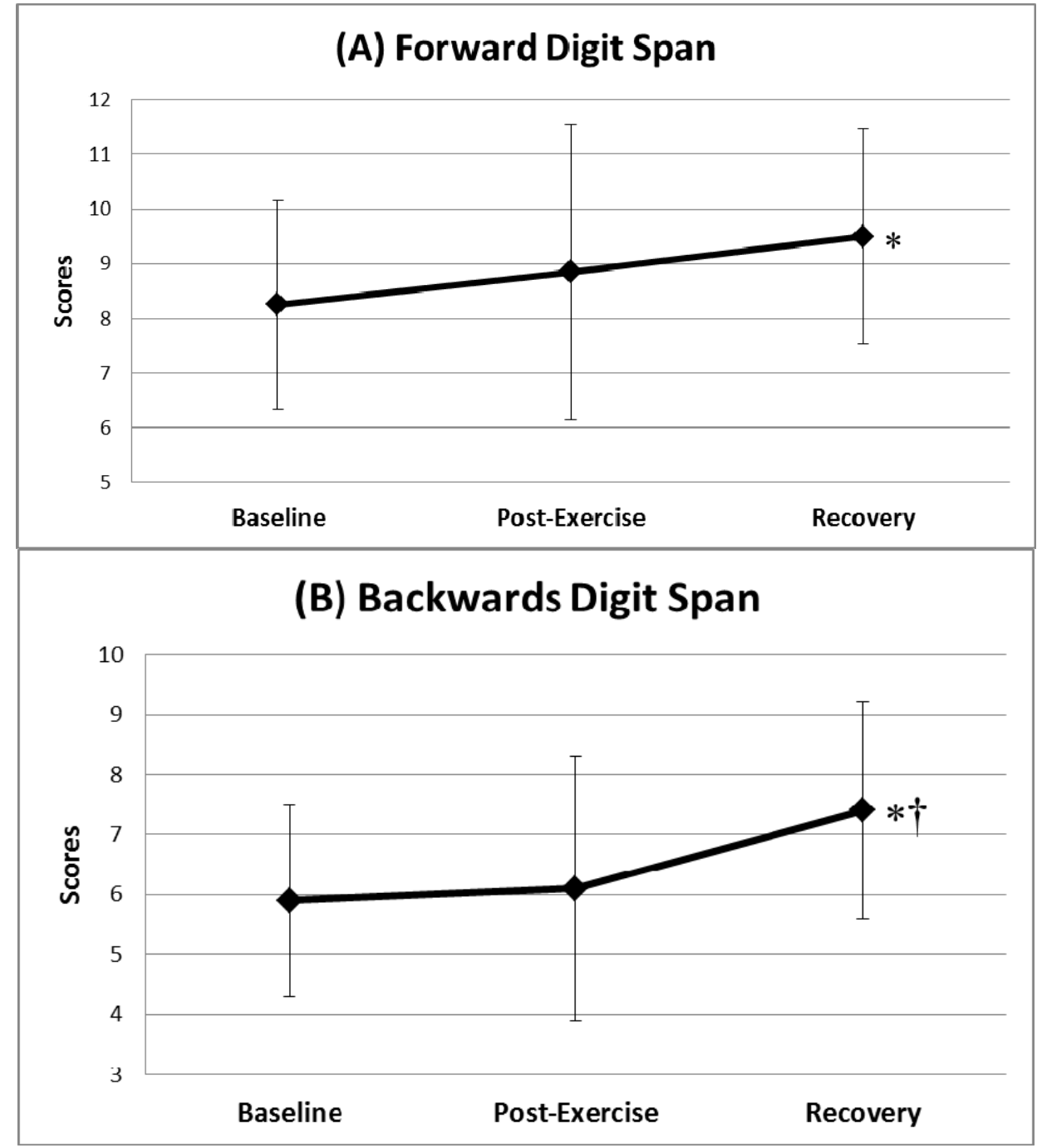

Figure 1

Mean test scores at baseline, after maximal intensity exercise, and after a one hour rest for (A) Forward Digit Span test and (B) Backward Digit Span test. *-differs significantly from baseline. t-differs significantly from post-exercise. Error bars are SD. 


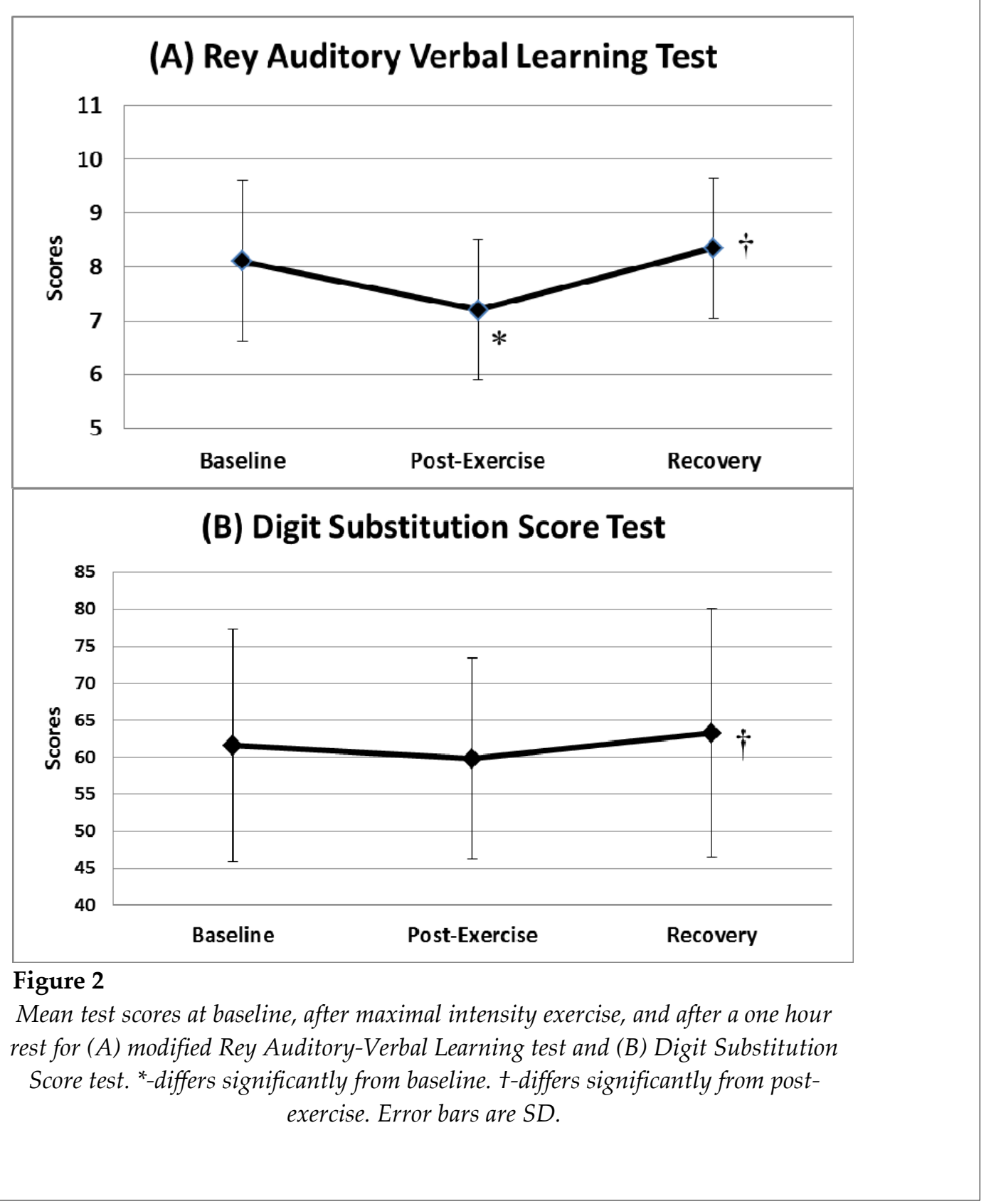

\section{Discussion}

The aim of this study was to examine the effects of acute maximal-intensity exercise on cognitive performance, for the first time in children and adolescents. Studies in adults indicated transient decrements following intense activity in cognitive processing, including working memory, verbal memory and attention (Covassin et al., 2007; Lo Bue-Estes et al., 2008; Moore et al., 2012). On the other hand, sufficient recovery from intense exercise may also result in enhanced working memory abilities (Lo Bue-Estes et al., 2008).

This study had two main findings. First, both forward and backward mean digit span scores increased following recovery from maximal exercise compared with baseline values. This finding suggests improvements in the attention span and verbal working memory after recovery. Second, mean RAVLT scores significantly decreased immediately following maximal exercise, and returned to baseline values after one hour of recovery. This finding represents transient 
impairment in verbal learning ability. There was also a significant rise in DSST test scores from immediately after exercise to after recovery, with no significant difference between baseline and recovery measurements.

The transient effects found only in the RAVLT scores can be interpreted in relation to the specific cognitive functions evaluated by each of the neuro-cognitive tests used in this study. The part of the RAVLT used herein evaluated immediate recall memory, which had been previously linked to concentration abilities and the attention span in children with ADHD (Pineda et al., 2007). The digit span evaluates the attention span as well as short term workingmemory, the part of memory involved in learning, language comprehension and short-term storage of information for executing complex tasks (Wechsler 2003; Vakil et al., 2009). The DSST assesses visual-motor coordination, short term memory, concentration and processing speed, along with the ability to learn a new task (Wechsler, 2003; Vakil et al., 2009). Even though both digit span (as in the Digit Span Test) and word span (as in the RAVLT) tasks involve short term working memory, it was suggested that the digit span and word span did not correlate unless the words on the list were low in imagibility (Martin and Saffran, 1999). The word span is more related to learning ability than the digit span, as the former engages more lexical and semantic processes (Martin and Saffran, 1999). A metaanalysis concerning the effects of acute exercise on cognitive performance reported a negative effect for the auditory verbal learning test and a nonsignificant effect for the forward digit span (Chang et al., 2012). Our findings, therefore, are in agreement with previous data obtained from adults (Covassin et al., 2007), suggesting that in children as well, maximal intensity exercise might lead to impairment in verbal learning. This effect may be the result of vascular changes in the brain that lead to cerebral fatigue (Shibuya et al., 2004a, b) as well as to an elevated heart rate that influences brain activity (Nishihira et al., 1999).

Our exercise protocol included performing a graded maximal exercise test to volitional fatigue, which lasted 10:15 minutes on average (Table 1). The duration of exercise had been previously found to moderate the effects of exercise on cognitive function (Chang et al., 2012;
Donnelly et al., 2016). Chang et al. (2012) reported that shorter exercise bouts negatively affected cognitive performance, but longer exercise bouts had positive effects. It appeared that at least 20 min of exercise were necessary to see beneficial effects. Yet, this analysis did not take into consideration the intensity of exercise. In the present study, all participants exercised for much lower duration than 20 minutes, and still demonstrated cognitive changes in both directions. Future research should attempt to standardize the duration of exercise and its intensity prior to cognitive testing, as well as examine the effects of over 20 minutes of high intensity exercise on children's cognitive function.

The improvements seen in the Forward and Backward Digit Span tests from baseline to after recovery could possibly be attributed to learning effects. However, a previous study found that hardly any learning effects occurred over 20 repeated administrations of these tests in healthy adults (Wilson et al., 2000). Therefore, we tend to attribute these improvements, as well as the rise in mean DSST scores, to delayed beneficial cognitive effects of high intensity exercise. A previous study conducted in female college students (Lo Bue-Estes et al., 2008) found increased cognitive performance following a 30 min recovery from acute high-intensity exercise. This positive effect was attributed to the physiological and metabolic changes occurring during exercise, coupled with the removal of cerebral and peripheral demands post exercise.

Our findings have several clinical implications, pertinent to academic activities as well as to sports performance. Schools for example, may consider the types of cognitive activities conducted immediately after a high intensity PA, as well as the potential advantage of a delayed response to such an activity. Ma et al. (2015) found positive effects of high-intensity, short interval exercises on children's selective cognition. Yet, these researchers used a 10-min delay from the end of the exercise to the onset of cognitive testing. In this context, it might be beneficial not to conduct language lessons immediately following a physical education session in which a competition or a fitness test was performed, especially if high fatigue was experienced. Alternatively, it might be better to either provide children with a substantial 
recovery period, or include an in-between session that does not require verbal learning skills or a significant attention span. The decrease in verbal learning ability and immediate recall memory found in this study are also of relevance in competitive sports. Memmert and Furley (2007) found that in young handball players, more tactical instructions led to a narrower breadth of attention, which resulted in decreased performance (i.e., passing the ball to an unmarked player). It is therefore possible that in high intensity sports that lead to fatigue, athletes' ability to effectively process complex verbal information is decreased due to attentional demands. Other examples of the need for verbal learning are receiving coaching instructions during breaks of high-intensity sports, such as basketball timeouts and between rounds in combat sports. Coaches should be aware of the potential decrease of verbal learning following high-intensity activity, refrain from giving complex verbal information, and perhaps rely more on simple instructions and non-verbal cues. Further research is required to determine the effects of fatigue on athletes' ability to process verbal information on the field or in the ring, and correctly execute verbal instructions.

As with all studies, we acknowledge that this study also had several limitations. First, we did not have a control group that completed the tests under resting conditions. Hence, additional possible explanations for the between-test changes could be learning effects or changes in participant motivation and stress levels following the test. We highlight that baseline scores were relatively similar to the norm scores, indicating that the participants did not differ much from the general population in their overall cognitive function. Based on the dynamics seen in previous research (Chang et al., 2012; Covassin et al., 2007; Lo Bue-Estes et al., 2008; Moore et al., 2012), we have grounds to attribute our findings to the intense exercise. Our limited sample size restricted us from addressing potential moderating factors such as the education level, pubertal status or physical fitness. Regarding the latter, we found no significant correlation between fitness levels and any of the tests examined, hence such analysis is not expected to materially affect our findings. Finally, the order of administration of the neuro-cognitive tests was fixed in order to achieve uniformity among participants. It is possible that administering the RAVLT or the DSST closer in time to exercise cessation may have produced different results, given the transient effects of maximal exercise on cognitive performance (Chang et al., 2012).

The effects of acute maximal-intensity exercise on cognitive performance in children should be further studied. Future studies should use other cognitive evaluations, perhaps in a mixed order, or preferably administer only one single test immediately after each exercise session. We also suggest comparing the effects of both moderate and vigorous exercise on the same child's cognitive performance. Finally, although a strength of our study was the inclusion of a one hour recovery stage, it is clinically useful to determine how long might it take for children to mentally recover from a maximal intensity exercise session, as well as gain potential cognitive increases. This can be achieved by conducting more post exercise testing closer to the completion of exercise (e.g., after $5 \mathrm{~min}$ and after $15 \mathrm{~min}$ ), and again, with only a single cognitive test.

In conclusion, this study was a novel attempt to examine the effects of acute maximal intensity exercise on cognitive performance in children. The study findings are in line with previous studies conducted with adults, indicating both debilitating (Covassin et al., 2007; Lo Bue-Estes et al., 2008; Moore et al., 2012) and facilitating (Lo Bue-Estes et al., 2008) effects for such an activity, depending on the cognitive task involved and the time elapsed from completion of exercise. Our findings suggest that a single bout of maximal intensity exercise in children can transiently impair cognitive performance involving complex tasks as verbal learning, which resolves after a one hour rest. On the other hand, more simple cognitive tasks that apply short term working memory are not negatively affected by such an activity, and may even be facilitated after sufficient rest. This positive effect complements the known beneficial effects of MVPA in children (Sibley and Etnier, 2003). Future studies are expected to shed additional light on the effects of maximal intensity exercise on cognitive performance in children, as well as the factors which moderate these effects. 


\section{References}

Alves CR, Tessaro VH, Teixeira LA, Murakava K, Roschel H, Gualano B, Takito MY. Influence of acute highintensity aerobic interval exercise bout on selective attention and short-term memory tasks. Percept Mot Skills, 2014; 118; 63-72

Best JR. Effects of physical activity on children's executive function: Contributions of experimental research on aerobic exercise. Dev Rev, 2010; 30: 331-551

Bongers BC, van Brussel M, Hulzebos EHJ, Takken T. Pediatric norms for cardiopulmonary exercise testing in relation to sex and age (2nd ed.). Hertogenbosch, the Netherlands: Uitgeverij BOXpress; 2014

Carlson SA, Fulton JE, Lee SM, Maynard LM, Brown DR, Khol HW, Dietz WH. Physical education and academic achievement in elementary school: Data from the early childhood longitudinal study. Am J Public Health, 2008; 98; 721-727

Chang YK, Labban JD, Gapin JI, Etnier JL. The effects of acute exercise on cognitive performance: A metaanalysis. Brain Res, 2012; 1453: 87-101

Covassin T, Weiss L, Powell J, Womack C. Effects of a maximal exercise test on neurocognitive function. $\mathrm{Br} J$ Sports Med, 2007; 41: 370-374

Davis CL, Tomporowski PD, McDowell JE, Austin BP, Miller PH, Yanasak NE, Allison JD, Naglieri JA. Exercise improves executive function and achievement and alters brain activation in overweight children: a randomized, controlled trial. Health Psychol, 2011; 30: 91-98

Diamond A. Executive functions. Annu Rev Psychol, 2013; 64: 135-168

Donelly JE, Hillman CH, Casteli D, Etnier JL, Lee S, Tomporowski P, Lambourne K, Szabo-Reed AN. Physical activity, fitness, cognitive function, and academic achievement in children: A systematic review. Med Sci Sports Exerc, 2016; 48:1197-222

Godfrey S. The response of normal children to exercise. In: Godfrey S, ed. Exercise Testing in Children. W.B. Saunders Company Ltd. London, UK, 66-101; 1974

Hillman CH, Pontifex MB, Castelli DM, Khan NA, Raine LB, Scudder MR, Drollette ES, Moore RD, Wu CT, Kamijo K. Effects of the FITKids randomized controlled trial on executive control and brain function. Pediatrics, 2014; 134: 1063-1071

Isaacs LD, Pohlman EL. Effects of exercise intensity on an accompanying timing task. J Hum Movement Stud, 1991; 20: 123-131

Lo Bue-Estes C, Willer B, Burton H, Leddy JJ, Wilding GE, Horvath PJ. Short-term exercise to exhaustion and its effects on cognitive function in young women. Percep Motor Skill, 2008; 107: 933-945

Ma JK, Le Mare L, Gurd BJ. Four minutes of in-class high-intensity interval activity improves selective attention in 9- to 11-year olds. Appl Physiol Nutr Metab, 2015; 40: 238-244

Martin N, Saffran EM. Effects of word processing and short-term memory deficits on verbal learning: Evidence from aphasia. Inter Jour Psych, 1999; 34: 339-346

McMorris T, Keen P. Effect of exercise on simple reaction times of recreational athletes. Percept Motor Skill, 1994; 78: 123-130

Memmert D, Furley D. “I spy with my little eye!": Breadth of attention, inattentional blindness, and tactical decision making in team sports. J Sport Exerc Psychol, 2007; 29: 365-381

Moore RD, Romine MW, O'Connor PJ, Tomporowski PD. The influence of exercise-induced fatigue on cognitive function. J Sports Sci, 2012; 30: 841-850

Nishihira Y, Ohno T, Hatta A, Fumoto M, Kaneda T, Tokitou S, Imanaka K, Funase K. P300 before and after transient hard exercise. Adv. Exerc. Sports Physiol, 1999; 5: 49-54

Physical Activity Guidelines Advisory Committee. Physical Activity Guidelines Advisory Committee Report, 2008. Washington, DC: U.S. Department of Health and Human Services; 2008

Pineda DA, Puerta IC, Aguirre DC, García-Barrera MA, Kamphaus RW. The role of neuropsychologic tests 
in the diagnosis of attention deficit hyperactivity disorder. Pediatr Neurol, 2007; 36: 373-381

Shibuya K, Tanaka J, Kuboyama N, Murai S, Ogaki T. Cerebral cortex activation during supramaximal exhaustive exercise. J. Sports Med. Phys. Fitness, 2004a; 44: 215-219

Shibuya K, Tanaka J, Kuboyama N, Murai S, Ogaki T. Cerebral oxygenation during intermittent supramaximal exercise. Respir Physiol Neurobiol, 2004b; 40: 165-172

Sibley BA, Etnier JL. The relationship between physical activity and cognition in children: a meta-analysis. Pediatr Exerc Sci, 2003; 15: 243-256

Singh A, Uijtdewilligen L, Twisk JW, van Mechelen W, Chinapaw MJ. Physical activity and performance at school: a systematic review of the literature including a methodological quality assessment. Arch Pediatr Adolesc Med, 2012; 166: 49-55

Strong WB, Malina RM, et al. Evidence based physical activity for school-age youth. J Pediatr, 2005; 146: 732737

Tomporowski PD, Lambourne K, Okumura MS. Physical activity interventions and children's mental function: An introduction and overview. Prev Med, 2011; 52S, S3-S9

Tomporowski PD, Davis CL, Miller PH, Naglieri JA. Exercise and children's intelligence, cognition, and academic achievement. Educ Psychol Rev, 2008; 20: 111-131

Tomporowski PD. Cognitive and behavioral responses to acute exercise in youth: A review. Pediatr Exerc Sci, 2003; 15: 348-359

Vakil E, Blachstein H, Sheinman M, Greenstein Y. Developmental changes in attention tests norms: Implications for the structure of attention. Child Neuropsychol, 2009; 15: 21-39

Vakil E, Blachstein H, Sheinman M. Rey AVLT: developmental normas for children and the sensitivity of different memory measures to age. Child Neuropsychol, 1998; 4: 161-177

Wechsler D. Wechsler Intelligence Scale for Children-4th Edition (WISCIV). San Antonio, TX: Harcourt Assessment; 2003

Wilson BA, Watson PC, Baddeley AD, Emslie H, Evans JJ. Improvement or simply practice? The effects of twenty repeated assessments on people with and without brain injury. J Int Neuropsychol Soc, 2000; 6: $469-479$

\section{Corresponding author:}

\section{Gal Dubnov-Raz MD MSc}

Exercise, Nutrition and Lifestyle Clinic, The Edmond and Lily Safra Children's Hospital, Sheba Medical

Center, Tel-Hashomer, Israel

Phone: +972-54-4570250, Fax: +972-3-5472503,

E-mail: gal.dubnov-raz@sheba.health.gov.il 